\title{
A Framework for Multidimensional Modelling of Spectrum Occupancy in the Simulation of Cognitive Radio Systems
}

\author{
Miguel López-Benítez \\ Dept. Electrical Engineering and Electronics \\ University of Liverpool, United Kingdom \\ Email: M.Lopez-Benitez@liverpool.ac.uk
}

\author{
Fernando Casadevall \\ Dept. Signal Theory and Communications \\ Universitat Politècnica de Catalunya, Spain \\ Email: ferranc@tsc.upc.edu
}

\begin{abstract}
Spectrum models play a key role in the research of Dynamic Spectrum Access/Cognitive Radio (DSA/CR) systems. Models existing to date are able to characterise the statistical properties of spectrum occupancy in only one of its dimensions (time, frequency or space). However, to the best of the authors' knowledge, there is no model capable to account for the properties of spectrum occupancy in all its dimensions simultaneously. Since the development of a single model to characterise spectrum occupancy in such a holistic manner constitutes an extremely challenging task, this work explores an alternative based on the combination of existing dimension-specific models into a unified modelling approach. In particular, a framework is proposed for the integration of spectrum models that have been designed independently for a specific dimension of spectrum occupancy (time, frequency or space), thus enabling the multidimensional modelling of spectrum occupancy. A sophisticated simulation tool implementing state-of-the-art time, frequency and space dimension models is developed according to the proposed framework and used to generate synthetic spectrum data that simultaneously accounts for the properties of spectrum occupancy in all its dimensions. The use of such modelling framework in the context of the simulation of DSA/CR systems is illustrated as well.
\end{abstract}

Keywords-Cognitive radio; dynamic spectrum access; spectrum models; multidimensional modelling; simulation.

\section{INTRODUCTION}

The Dynamic Spectrum Access (DSA) principle [1-4], based on the Cognitive Radio (CR) paradigm [5-9], is aimed at increasing the efficiency of spectrum utilisation by allowing unlicensed (secondary) users to access opportunistically what is known as white spaces or spectrum holes [10], i.e., spectrum gaps in licensed bands temporarily and/or spatially unused by the licensed (primary) users. Owing to the opportunistic nature of this spectrum access paradigm, the behaviour and performance of DSA/CR systems is highly dependent on the spectrum occupancy pattern of primary users. Thus, spectrum occupancy models are of paramount importance in DSA/CR research. Spectrum models can find many relevant applications ranging from analytical studies to the design and dimensioning of secondary networks, as well as the development of innovative simulation tools and more efficient DSA techniques.

A wide range of spectrum occupancy models has been developed to characterise and reproduce the statistical properties of spectrum occupancy in the time, frequency and space dimensions. Given the complexity of spectrum modelling, existing models have normally focused on the characterisation of statistical properties in one specific dimension (i.e., time, frequency or space). While existing models are certainly useful in DSA/CR research, they only enable the study and analysis of spectrum occupancy patterns and their statistical properties in only one of the aforementioned dimensions. In order to characterise spectrum occupancy in a holistic manner, the simultaneous modelling of the statistical properties in the time, frequency and space dimensions would be desirable. However, to the best of the authors' knowledge, the spectrum occupancy models existing to date do not enable such a holistic characterisation of spectrum occupancy. Modelling not only the time evolution (time dimension) of the occupancy of a group of channels within a frequency band (frequency dimension) but also how it is perceived by DSA/CR users at different locations as a function of the radio propagation environment (space dimension) is an extremely challenging task. Given the inherent complexity of developing a single model that simultaneously characterises the statistical properties of spectrum occupancy in all its dimensions, a natural question is whether existing dimension-specific models could be combined in order to enable a multidimensional modelling of spectrum occupancy that simultaneously accounts for time, frequency and space dimension properties. This question motivates this work, where a framework is proposed for the integration of spectrum models that have been designed independently for a specific dimension of spectrum occupancy (time, frequency or space) into a unified modelling approach, thus enabling the multidimensional modelling of spectrum occupancy.

First, an overview of existing spectrum occupancy models for the time (Section II), frequency (Section III) and space (Section IV) dimensions is provided. The provided overview focuses on relevant parameters and properties that existing models are able to characterise and reproduce (a broader overview can be found in $[11,12]$ and a more detailed description and discussion is provided in [13, 14]). A framework for the integration of such models into a multidimensional modelling approach is then presented in Section V. In order to illustrate the use of the presented framework, selected time, frequency and space dimension models are implemented and integrated (according to the proposed framework) in a sophisticated simulation tool. Section VI discusses the use of the developed tool in the context of DSA/CR simulation to generate synthetic spectrum data that simultaneously accounts for the properties of spectrum occupancy in all its dimensions. Finally, Section VII summarises and concludes the paper. 


\section{TIME-DIMENSION MODELS}

The occupancy pattern of a primary channel in the time domain can be characterised in terms of three main aspects, namely the channel load or occupancy level (duty cycle), the durations of the busy/idle periods and the correlation structures of the period durations. These three aspects are discussed in more detail in the following.

A) Channel load (duty cycle): The channel load is a relevant parameter since it indicates the degree to which a channel may become available to a DSA/CR system for opportunistic use. The channel load can be characterised in terms of the duty cycle, which represents the probability or fraction of time that the channel is busy. While the average value of this parameter is relevant, its temporal variation pattern also represents an interesting aspect to characterise. In some cases, the channel duty cycle has been observed to follow a predominantly deterministic pattern as a result of the social behaviour and common habits of users (for example, this is a common case in cellular mobile communication systems [15]). While the use of time series analysis and AutoRegressive Integrated Moving Average (ARIMA) has been proposed in order to characterise the duty cycle pattern in this case [16], a deterministic modelling approach by means of tailored mathematical functions as proposed in [17] can be simpler (the computation of the duty cycle value at a particular time instant is direct and does not require the computation of past values at previous time instants as it is the case of time series) and can provide satisfactory results as well. However, in some other cases the variation pattern of the channel duty cycle may exhibit a highly random component as a result of a number of random factors (e.g., instantaneous number of active users, resource management policies, etc.), which may lead to highly unpredictable variations [18]. In such a case, a stochastic modelling approach as the one proposed in [17] becomes a more convenient approach.

B) Period durations: A complete and realistic modelling of a channel requires not only the duty cycle to be taken into account but also the durations of busy and idle periods and their underlying distributions. A common assumption in existing literature is that period durations are exponentially distributed, which enables the simplified modelling of the on/off pattern of a channel as a two-state Continuous-Time Markov Chain (CTMC) model (with two states used to represent the busy and idle states of the channel) where the sojourn times/state holding times (i.e., the period durations) are modelled as exponentially distributed random variables. However, various field measurements have demonstrated that period durations are not exponentially distributed in real systems [19-23]. Period durations have been found to be more adequately described by means of generalised Pareto [19], a mixture of uniform and generalised Pareto [20, 21], hyper-Erlang [20, 21], generalised Pareto and hyper-exponential [22] as well as geometric and log-normal [23] distributions. A more detailed and in-depth study on the best-fitting distribution for various radio technologies, taking into account both short-term and long-term time scales, can be found in [24]. Based on the conclusions from empirical modelling works, a more appropriate model is therefore the Continuous-Time Semi-Markov Chain (CTSMC) model, where the state holding times can follow any arbitrary (not necessarily exponential) distribution.
C) Correlation properties of period durations: The durations of busy and idle periods can be correlated [23]. Two different types of correlation can be distinguished. The first type is the correlation between the durations of periods of different type (i.e., correlation of busy and idle periods), which can be modelled simply by the corresponding correlation coefficients, which have been shown to take negative values [25] (i.e., when the duration of a busy period increases, the duration of the next idle period decrease and vice versa). The second type is the correlation between the sequence of periods of the same type (either busy or idle) of a channel and a shifted version of itself (i.e., the autocorrelation function), which has experimentally been observed to exhibit two different behaviours (periodic/oscillating and non-periodic/decreasing), for which appropriate mathematical functions have been proven to be a convenient modelling approach [25].

\section{FREQUENCY-DIMENSION MODELS}

Several aspects and parameters can be considered in the modelling of the occupancy pattern of a primary band in the frequency domain. Some examples of relevant parameters in the frequency domain include the statistical distribution of the duty cycle values for channels within the same band, and the clustering of the duty cycle over frequency. These aspects are discussed in more detail in the following.

A) Statistical distribution of duty cycle: The distribution of duty cycle values for individual channels within the same spectrum band has been shown to be accurately modelled by means of a beta distribution [26, 27]. The Kumaraswamy distribution, which closely resembles the beta distribution, can also be used as an appropriate model for the distribution of duty cycle values [27].

B) Clustering of duty cycle: Within a spectrum band, channels with similar load levels (i.e., duty cycle values) are not isolated but found in groups/clusters of a certain size, meaning that the duty cycle is clustered in the frequency domain [23, 27]. The proposed approaches for characterising this aspect are based on the definition of a set of duty cycle archetypes, with each archetype representing a range of duty cycle values. Based on such definition, a cluster can then be thought of as a group of channels that belong to the same duty cycle archetype (i.e., whose duty cycle values are all comprised within the same interval and therefore are similar). Following this approach, and according to experimental results, the cluster size (i.e., the number of channels within a cluster) can then be modeled as a random variable that follows a lognormal [23] or geometric [23, 27] distribution.

\section{SPACE-DIMENSION MODELS}

Spectrum models in the space domain are aimed at describing the spectrum occupancy perceived by DSA/CR users as a function of the user location, which can be characterised in various ways. A simple modelling approach commonly used in the existing literature is to determine the average power received from the primary transmitter at the DSA/CR user location (by means of path loss models) and compare to a decision threshold in order to determine if the DSA/CR user observes the sensed spectrum as busy or idle (this modelling approach assumes that DSA/CR users decide on the spectrum occupancy state based on energy detection [28, 29]). This 
binary busy/idle modelling approach can be complemented with spatial statistics by fitting an analytic semivariogram model to the predicted power levels, which permits reproducing some statistical properties (e.g., spatial correlations) of the powers observed over a certain region [30]. While such binary busy/idle modelling approaches may be attractive because of their simplicity, this kind of methods in general results in an oversimplified characterisation of the perceived occupancy (i.e., the spectrum at a given location is observed by DSA/CR users either as always busy or always idle). In practice, radio propagation effects (e.g., shadowing or multipath fading) may lead to momentary signal fades that result in signal misdetections (i.e., the spectrum may not be observed in practice as always busy) or the receiver noise may lead to false alarms (i.e., the spectrum may not be observed in practice as always idle) [31]. Based on this appreciation, the probability to observe the spectrum as busy constitutes a more appropriate modelling parameter to characterise the spectrum occupancy perceived at various locations. In the modelling approach presented in [32], the power levels predicted by the propagation (path loss) model are not directly compared to a threshold but mapped (by means of the duty cycle model proposed in [33], which implicitly accounts for shadowing and multipath fading) to the probability that the spectrum is perceived as busy by DSA/CR users. This probability can be computed individually for each location of interest but can also be correlated with the simultaneous observations of other users at different locations by means of the model for concurrent observations proposed in [13]. This probabilistic modelling approach is more complex than the binary approach discussed above, but provides a more realistic characterisation of the spectrum occupancy pattern observed in the space domain.

\section{FRAMEWORK FOR MULTIDIMENSIONAL MODELLING}

As it can be appreciated in the overview provided in Sections II, III and IV, spectrum models existing to date are dimension-specific and therefore capable of characterising the statistical properties of spectrum occupancy in only one of its dimensions (time, frequency or space), but not in all its dimensions simultaneously. To cope with this limitation, this section proposes a framework for the integration of spectrum models that have been designed independently for a specific dimension of spectrum occupancy (time, frequency or space) into a unified modelling approach, thus enabling a multidimensional modelling of spectrum occupancy that simultaneously accounts for time, frequency and space dimension properties.

An important field of application of spectrum occupancy models is the development of simulation tools for the performance evaluation of DSA/CR networks and their associated techniques. The proposed framework is envisaged in the context of simulation of DSA/CR systems, bearing in mind as a main application the generation of synthetic spectrum occupancy data for simulation purposes (other applications can be found). In particular, this section provides a detailed description of how existing dimension-specific models can be combined and used together in order to generate artificial spectrum data capable to reproduce the statistical properties of spectrum occupancy in the time, frequency and space dimensions. Some aspects of the modelling framework proposed in this section are based on arbitrary decisions but can easily be adapted in order to meet particular simulation needs.

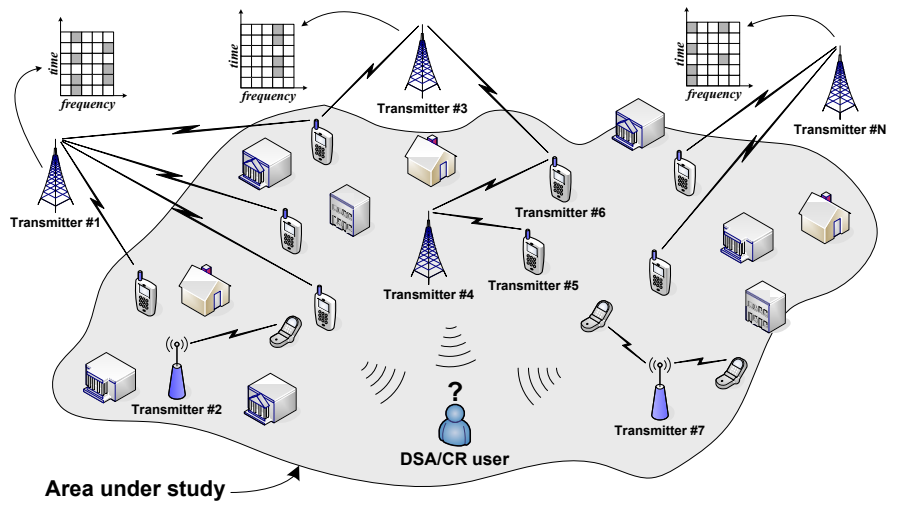

Fig. 1. Generic simulation scenario [14].

A generic simulation scenario is shown in Figure 1. The simulation scenario considers a specified geographical area where secondary DSA/CR users try to exploit the spectrum opportunities (spectrum holes/white spaces) of a number of primary transmitters whose coverage areas overlap (totally or partially) with the considered geographical area under study. Primary transmitters may be located inside or outside the geographical area under study and may be fixed or mobile. A mobile primary transmitter would imply that its location needs be recomputed periodically during the simulation according to an appropriate mobility model (e.g., see [34]). Each primary transmitter operates over a number of licensed radio channels leading to a particular time-frequency transmission pattern as illustrated in Figure 1. The time-frequency pattern of each primary transmitter is defined by the set of radio channels over which the transmitter operates, the binary busy/idle occupancy sequence of each radio channel as well as the employed transmission powers. Note that the transmission power may not be constant (e.g., in the case of a time-slotted downlink channel where various slots are allocated to various primary receivers at different locations requiring different transmission powers). Considering this scenario, the objective is to generate the timefrequency transmission patterns for the primary transmitters involved in the scenario and determine how they would be perceived by a DSA/CR user located anywhere within the geographical area under study (note that a time-frequency transmission pattern may be observed in different ways depending on the actual location of the DSA/CR observer).

The framework proposed for the generation of multidimensional artificial spectrum data based on dimension-specific models is illustrated in Figure 2. The whole process is divided into two stages: a first stage where a Global Time-Frequency Map (GTFM) is generated (based on time and frequency models) for each primary transmitter, and a second stage where Local Time-Frequency Maps (LTFMs) are generated (based on space models) for each DSA/CR user. A GTFM represents the actual time-frequency transmission pattern of a primary transmitter, while an LTFM represents the way a GTFM is perceived/observed by a DSA/CR user at a specific location within the area under study. Therefore, for a single GTFM, many LTFMs need to be generated as shown in Figure 2.

A GTFM could be generated by simply generating an on/off (busy/idle) sequence for each channel independently, which can be accomplished by means of appropriate timedimension models. However, the occupancy patterns of a 

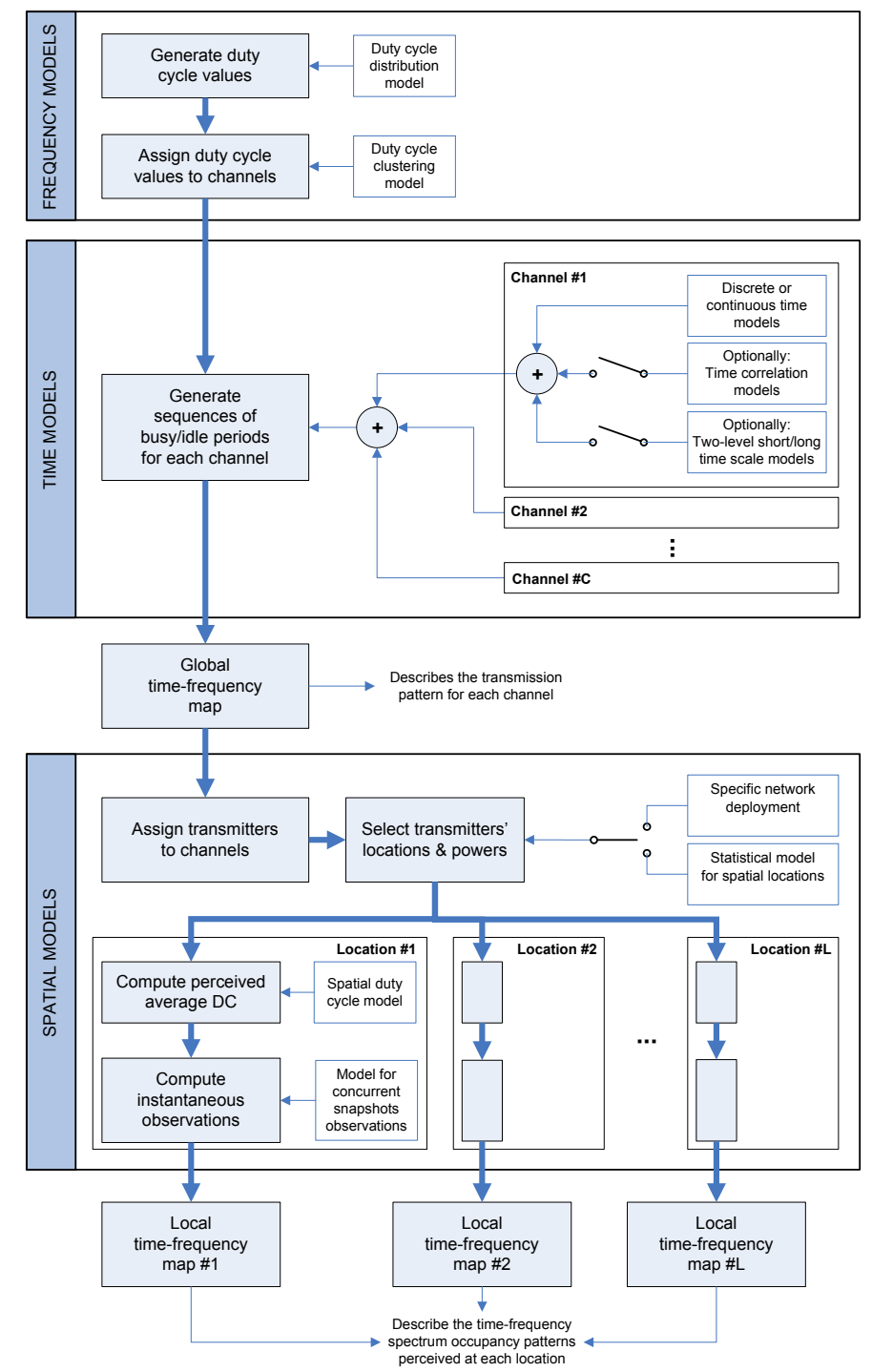

Fig. 2. Proposed framework for multidimensional modelling [14].

group of channels within the same spectrum band are not completely independent and unrelated. In order to account for this, frequency-dimension models are used in the first place. The first step is to generate a set of duty cycle values, one for each channel in the GTFM, which can be obtained as random numbers drawn from a beta or Kumaraswamy distribution as detailed in Section III-A. These duty cycle values need to be assigned to the channels in the GTFM. A random allocation of duty cycle values to channels would not be able to reproduce the clustering of the duty cycle over frequency (i.e., the existence of groups of channels with similar duty cycle values) as discussed in Section III-B. In order to take into account the clustering properties observed in real systems, the algorithm proposed in [27] can be used, which performs such allocation so that the duty cycle clustering effect is reproduced.

Once the duty cycle values have been allocated to the radio channels in the GTFM (taking into account the appropriate duty cycle distribution and clustering as discussed above), the on/off (busy/idle) sequence for each channel can be generated. As discussed in Section II, the generated sequences need to reproduce not only the average duty cycle value assigned to each channel (Section II-A) but also the distribution of period durations for both idle and busy periods (Section II-B). In the case of discrete-time simulations, this can be accomplished by implementing the model presented in [17], which is based on the use of a Markov chain along with appropriate duty cycle models. In the case of continuous-time simulations, this can be accomplished by choosing an appropriate distribution for the period durations (this depends on the particular radio technology of the primary transmitter, see [24] for a detailed list of distributions for various radio technologies) and then configuring the distribution parameters in such a way that the target duty cycle is met (the duty cycle of a channel is $\mathbb{E}\left\{T_{1}\right\} /\left(\mathbb{E}\left\{T_{0}\right\}+\mathbb{E}\left\{T_{1}\right\}\right)$, where $\mathbb{E}\left\{T_{0}\right\}$ and $\mathbb{E}\left\{T_{1}\right\}$ are the mean value of idle and busy periods, respectively, and the relation between the distribution parameters and the mean value depends on the distribution - see Table I in [24]). Once that appropriate distributions are chosen for the busy/idle durations of a channel, and configured in order to reproduce the assigned duty cycle value, the period durations can simply be obtained as random numbers drawn from such distributions. Alternatively, the period durations can also be generated following the slightly more complex algorithm proposed in [25], which allows reproducing not only specified average duty cycles and distributions of period durations, but also correlation properties among period durations, which is another important aspect as discussed in Section II-C. Moreover, the two-level modelling approaches proposed in [24] can also be incorporated in order to induce specific temporal characteristics in the short- and long-term channel occupancy patterns (depending on the considered radio technology, see [24] for details). After applying the aforementioned steps, the final result is a GTFM consisting of a time-domain binary on/off occupancy sequence for each of the radio channels in the GTFM, which implicitly reproduces the relevant properties of spectrum occupancy in the time and frequency domains as modelled by the individual dimension-specific models.

The purpose of the second stage of the proposed framework is to translate the GTFM obtained in the first stage (actual/real time-frequency transmission pattern) into a set of LTFMs (perceived/observed time-frequency occupancy patterns) for specific DSA/CR user locations within the area under study. To this end, several considerations along with appropriate space models are applied as detailed in the following.

The first step of the second stage is to decide the primary transmitter(s) associated to each radio channel of the GTFM along with the corresponding location(s) and transmission power(s). In the discussion provided above, a single primary transmitter has been assumed for the GTFM (i.e., the activity in the GTFM is assumed to be the result of a single primary transmitter). However, it is also possible to assume multiple primary transmitters within a single GTFM (where each primary transmitter operates over a subset of radio channels in the GTFM). The reason for including the possibility of multiple primary transmitters in a single GTFM is to enable the proposed framework to realistically reflect the situation where several primary transmitters operate within the spectrum of interest for the DSA/CR system (for example, if the primary system is based on a cellular structure). The spectral activity reflected in the GTFM and the way it is generated in the first stage of the framework is not affected by the fact of 
considering that the GTFM is the result of a single or multiple primary transmitters. However, this is important when computing the LTFMs because certain properties of the primary transmitters (locations, transmission powers, etc.) determine how much power will be received by a DSA/CR user at a particular location and therefore the way spectrum occupancy will be perceived by the DSA/CR user. This means, when computing the LTFMs based on a GTMF, that each subset of radio channels in the GTFM will need to be processed with different parameters depending on the primary transmitter that is considered to operate over the subset (taking into account the location, transmission power and other relevant aspects of each primary transmitter). The locations, transmission powers and other relevant parameters of the primary transmitters can be selected individually so as to reproduce specific well-defined network deployments or making use of statistical models for spatial locations, which are out of the scope of this work.

After deciding the transmitter-to-channel assignments, the next step is to determine how the GTFM is perceived by DSA/CR users at arbitrary locations within the area under study (i.e., the LTFMs). This can be accomplished by means of the spatial simulation method described in [13]. In order to employ this simulation approach, it is necessary to compute first the average duty cycle perceived at each DSA/CR user location for each channel in the GTFM (i.e., each channel has an average duty cycle, but the average duty cycle perceived at each location may be different as a result of radio propagation). The average duty cycle observed at each DSA/CR user location can be computed by means of the spatial duty cycle model proposed in [33] along with the statistical prediction method proposed in [32]. The model for concurrent snapshot observations proposed in [13] can then be applied. This model takes into account the set of conditional probabilities $P\left(s_{i} \mid s_{i}^{*}\right)$ to observe a channel in state $s_{i}$ at any arbitrary location given that it is observed in state $s_{i}^{*}$ at a reference location where the SNR is maximum (i.e., greater than the SNR at any other location within the area under study), with $i=0$ and $i=1$ denoting idle and busy states, respectively (these conditional probabilities are computed based on the observed duty cycles computed in the previous step). For the application of such model, the reference location for each channel in the GTFM needs to be selected as the location of the primary transmitter (i.e., where the SNR is maximum). The LTFM for each DSA/CR user location can then be determined based on the conditional probabilities as follows: whenever the state of a channel in the GTFM is busy $\left(s_{1}^{*}\right)$, the channel in the LTFM is observed as busy $\left(s_{1}\right)$ with probability $P\left(s_{1} \mid s_{1}^{*}\right)$ (i.e., probability of detection), and whenever the state of a channel in the GTFM is idle $\left(s_{0}^{*}\right)$, the channel in the LTFM is observed as busy $\left(s_{1}\right)$ with probability $P\left(s_{1} \mid s_{0}^{*}\right)$ (i.e., probability of false alarm). This procedure reproduces not only the effect of radio propagation (i.e., signal missed detections) but also the effect of imperfect receivers (i.e., false alarms). Following this procedure, the GTFM can be extrapolated to the LTFM observed at any arbitrary location within the area under study.

The proposed framework combines individual dimensionspecific spectrum models to generate LTFMs that simultaneously reproduce statistical properties of spectrum occupancy in the time, frequency and space dimensions, thus enabling a multidimensional modelling of spectrum occupancy that simultaneously accounts for relevant properties in all its dimensions.

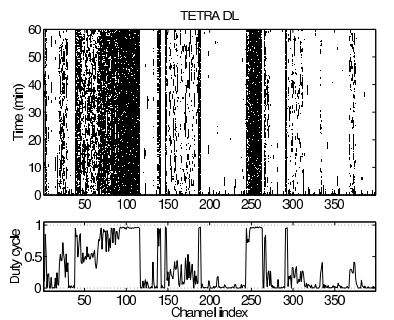

(a)

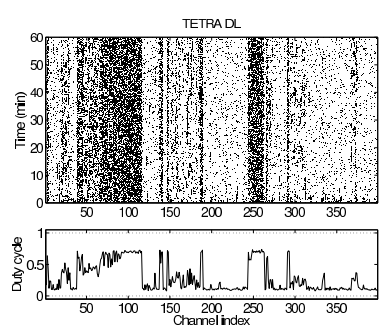

(c)

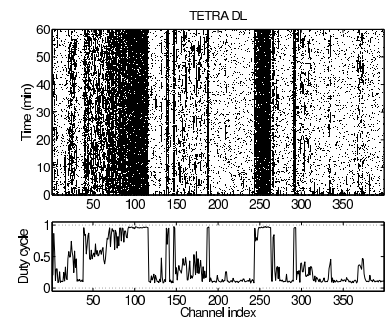

(b)

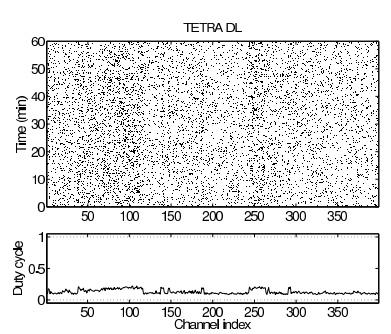

(d)
Fig. 3. Examples of synthetic spectrum occupancy data [14]: (a) GTFM, (b) LTFM at $10 \mathrm{~dB}$, (c) LTFM at $3 \mathrm{~dB}$, (d) LTFM at $0 \mathrm{~dB}$.

\section{ILLUSTRATIVE EXAMPLES}

In order to illustrate the presented framework, a sophisticated simulation tool was developed by integrating (according to the proposed framework) selected models of spectrum occupancy, enabling the reproduction of statistical properties in the time dimension (channel duty cycle and distribution of period durations [24] along with time correlations [25]), frequency dimension (distribution and clustering of channel duty cycles [27]) and space dimension (spatial dependence of the observed channel duty cycle [32, 33] and concurrent observations of the instantaneous channel state [13]).

The implemented simulation tool was used to generate synthetic spectrum data. Figure 3 shows as an example the GTFM and the corresponding LTFMs observed at a SNR of $10 \mathrm{~dB}, 3 \mathrm{~dB}$ and $0 \mathrm{~dB}$ (upper part of each figure) along with the corresponding duty cycle distribution over frequency (lower part of each figure) for a TETRA downlink system. A black (white) dot indicates a channel that is busy (idle) at that moment in the GTFM, or observed as such in the LTFMs.

As appreciated in Figure 3(a), the first stage of the proposed framework generates a GTFM that reproduces not only the on/off sequence of each individual channel in the time domain but also the statistical properties in the frequency domain (the duty cycle clustering can be clearly appreciated). In the second stage, the GTFM is extrapolated to the local perceptions of DSA/CR users at different locations (i.e., the LTFMs), experiencing different radio propagation conditions (i.e., different SNR values). As shown in Figure 3(b), under high SNR conditions the channels are observed as busy whenever they are actually busy. However, there is an appreciable number of points indicating that, in some cases, the channel is detected as busy when it is actually idle. These points correspond to false alarms where the noise power of the receiver surpasses the decision threshold. In fact, while the duty cycle shown in Figure 3(a) takes values within the interval $[0,1]$, in Figure 3(b) it is above $10 \%$ for all channels (this is due to the target false alarm rate of $10 \%$ selected to set the sensing decision threshold 
of DSA/CR users). The dependency of the observed spectrum occupancy with the DSA/CR user location can be observed by comparing the LTFMs obtained at different SNR values. For locations where the experienced SNR is low, the signal of several channels may be received below the sensing decision threshold (i.e., it is not detected). This is clearly shown in Figure 3(c), where the perceived duty cycle values are notably lower than in Figure 3(b). At locations with very low SNR, as in the example of Figure 3(d), signals can hardly be detected and the observed busy states are due mostly to false alarms (observed duty cycles are similar to the target false alarm rate).

It is worth noting in the examples of Figures 3(b), 3(c) and $3(\mathrm{~d})$ that all channels experience a similar reduction of the duty cycle. This is due to the consideration of a single primary transmitter in the generated GTFM, which results in the same SNR increase/reduction for all radio channels (and thus in a similar duty cycle variation) when the considered DSA/CR user location changes. In a configuration where the radio channels in the GTFM belong to different primary transmitters at different locations, a displacement of the DSA/CR user would result in approaching or moving away from various transmitters and hence different SNR increases/reductions for each individual radio channel. However, a simple configuration has been selected here for simplicity and clarity reasons.

\section{CONCLUSIONS}

Spectrum occupancy models play a key role in the research of DSA/CR systems. Existing models are dimension-specific (i.e., they reproduce the statistical properties of spectrum occupancy in only one dimension: time, frequency or space). This work has proposed a framework that combines individual dimension-specific spectrum models in order to simultaneously reproduce statistical properties of spectrum occupancy in the time, frequency and space dimensions, thus enabling a multidimensional modelling of spectrum occupancy.

\section{REFERENCES}

[1] C. Jackson, "Dynamic sharing of radio spectrum: A brief history," in Proc. First IEEE Int'l. Symp. Dyn. Spect. Access Networks (DySPAN 2005), Nov. 2005, pp. 445-466.

[2] Q. Zhao and A. Swami, "A survey of dynamic spectrum access: signal processing and networking perspectives," in Proc. IEEE Int'l. Conf. Acoustics, Speech and Signal Process. (ICASSP 2007), vol. 4, Apr. 2007, pp. IV/1349-IV/1352.

[3] Q. Zhao and B. M. Sadler, "A survey of dynamic spectrum access," IEEE Signal Process. Mag., vol. 24, no. 3, pp. 79-89, May 2007.

[4] M. M. Buddhikot, "Understanding dynamic spectrum access: Taxonomy, models and challenges," in Proc. 2nd IEEE Int'l. Symp. Dyn. Spect. Access Networks (DySPAN 2007), Apr. 2007, pp. 649-663.

[5] J. Mitola and G. Q. Maguire, "Cognitive radio: making software radios more personal," IEEE Pers. Comms., vol. 6, no. 4, pp. 13-18, Aug. 1999.

[6] J. Mitola, Cognitive Radio Architecture. Wiley-Interscience, Oct. 2006.

[7] S. Haykin, "Cognitive radio: Brain-empowered wireless communications," IEEE J. Sel. Areas Comms., vol. 23, no. 2, pp. 201-220, Feb. 2005 .

[8] Y.-C. Liang, K.-C. Chen, G. Y. Li, and P. Mähönen, "Cognitive radio networking and communications: An overview," IEEE Trans. Vehic. Tech., vol. 60, no. 7, pp. 3386-3407, Sep. 2011.

[9] M. López-Benítez, "Cognitive radio," in Heterogeneous cellular networks: Theory, simulation and deployment. Cambridge University Press, 2013, ch. 13, pp. 383-425.

[10] R. Tandra, A. Sahai, and S. M. Mishra, "What is a spectrum hole and what does it take to recognize one?" Proc. IEEE, vol. 97, no. 5, pp. 824-848, May 2009.

[11] M. López-Benítez and F. Casadevall, "Spectrum usage in cognitive radio networks: From field measurements to empirical models," IEICE Trans.
Comms., Special Section on Technologies for Effective Utilization of Spectrum White Space, vol. E97-B, no. 2, pp. 1-9, Feb 2014.

[12] $\_$,An overview of spectrum occupancy models for cognitive radio networks," in Proc. IFIP 6th Int'l. Conf. Netw. (NETWORKING 2011), May 2011, pp. 32-41.

[13] M. López-Benítez, "Spectrum usage models for the analysis, design and simulation of cognitive radio networks," Ph.D. dissertation, Dept. Signal Theory and Comms., Universitat Politècnica de Catalunya, 2011.

[14] M. López-Benítez and F. Casadevall, "Spectrum usage models for the analysis, design and simulation of cognitive radio networks," in Cognitive radio and its application for next generation cellular and wireless networks. Springer, 2012, ch. 2, pp. 27-73.

[15] D. Willkomm, S. Machiraju, J. Bolot, and A. Wolisz, "Primary user behavior in cellular networks and implications for dynamic spectrum access," IEEE Comms. Mag., vol. 47, no. 3, pp. 88-95, Mar. 2009.

[16] Z. Wang and S. Salous, "Spectrum occupancy statistics and time series models for cognitive radio," J. Sig. Proc. Systs., vol. 62, no. 2, pp. 145155, Feb. 2011.

[17] M. López-Benítez and F. Casadevall, "Empirical time-dimension model of spectrum use based on a discrete-time Markov chain with deterministic and stochastic duty cycle models," IEEE Trans. Vehic. Tech., vol. 60, no. 6, pp. 2519-2533, 2011.

[18] V. Blaschke, H. Jaekel, T. Renk, C. Kloeck, and F. K. Jondral, "Occupation measurements supporting dynamic spectrum allocation for cognitive radio design," in Proc. 2nd Int'l. Conf. Cognitive Radio Oriented Wireless Netws. \& Comms. (CrownCom 2007), Aug. 2007, pp. 50-57.

[19] S. Geirhofer, L. Tong, and B. M. Sadler, "A measurement-based model for dynamic spectrum access in WLAN channels," in Proc. IEEE Mil. Comms. Conf. (MILCOM 2006), Oct. 2006, pp. 1-7.

[20] _ - "Dynamic spectrum access in WLAN channels: Empirical model and its stochastic analysis," in Proc. 1st Int'l. Workshop Tech. Pol. \& Access. Spect. (TAPAS 2006), Aug. 2006, pp. 1-10.

[21] — - "Dynamic spectrum access in the time domain: Modeling and exploiting white space," IEEE Comms. Mag., vol. 45, no. 5, pp. 66-72, May 2007.

[22] L. Stabellini, "Quantifying and modeling spectrum opportunities in a real wireless environment," in Proc. IEEE Wireless Comms. \& Netw. Conf. (WCNC 2010), Apr. 2010, pp. 1-6.

[23] M. Wellens, J. Riihijärvi, and P. Mähönen, "Empirical time and frequency domain models of spectrum use," Phys. Comm., vol. 2, no. 1-2, pp. 10-32, Mar. 2009.

[24] M. López-Benítez and F. Casadevall, "Time-dimension models of spectrum usage for the analysis, design and simulation of cognitive radio networks," IEEE Trans. Vehic. Tech., vol. 62, no. 5, pp. 2091-2104, Jun. 2013.

[25] - "Modeling and simulation of time-correlation properties of spectrum use in cognitive radio," in Proc. 6th Int'l. ICST Conf. on Cognitive Radio Oriented Wireless Netws. (CrownCom 2011), Jun. 2011, pp. 1-5.

[26] M. Wellens and P. Mähönen, "Lessons learned from an extensive spectrum occupancy measurement campaign and a stochastic duty cycle model," Mobile Netws. \& Apps., vol. 15, no. 3, pp. 461-474, Jun. 2010.

[27] M. López-Benítez, F. Casadevall, D. López-Pérez, and A. V. Vasilakos, "Modeling and simulation of joint time-frequency properties of spectrum usage in cognitive radio," in Proc. 4th Int'l. Conf. Cognitive Radio and Adv. Spect. Management (CogART 2011), Oct. 2011, pp. 1-5.

[28] H. Urkowitz, "Energy detection of unknown deterministic signals," Proc. IEEE, vol. 55, no. 4, pp. 523-531, April 1967.

[29] T. Yücek and H. Arslan, "A survey of spectrum sensing algorithms for cognitive radio applications," IEEE Communications Surveys and Tutorials, vol. 11, no. 1, pp. 116-130, First Quarter 2009.

[30] M. Wellens, J. Riihijärvi, and P. Mähönen, "Spatial statistics and models of spectrum use," Computer Comms., vol. 32, pp. 1998-2001, Aug. 2009.

[31] M. López-Benítez and F. Casadevall, "On the spectrum occupancy perception of cognitive radio terminals in realistic scenarios," in Proc. 2nd Int'l. Workshop Cog. Inf. Proc. (CIP 2010), Jun. 2010, pp. 1-6.

[32] - "Statistical prediction of spectrum occupancy perception in dynamic spectrum access networks," in Proc. IEEE Int'l. Conf. Comms. (ICC 2011), Jun. 2011, pp. 1-6.

[33] _ "Spatial duty cycle model for cognitive radio," in Proc. 21st IEEE Int'l. Symp. Pers., Indoor and Mobile Radio Comms. (PIMRC 2010), Sep. 2010, pp. 1631-1636.

[34] M. López-Benítez, M. C. Lucas-Estañ, and J. Gozálvez, "A dynamic radio simulation platform for the study of radio resource management techniques in heterogeneous wireless systems," in Proc. 9-th ACM/IEEE Int'l. Symp. Modeling, Analysis and Simulation of Wireless and Mobile Systems (MSWiM 2006), Oct. 2006, pp. 143-171. 